RADIO SCIENCE, VOL. 41, RS4004, doi:10.1029/2005RS003307, 2006

\title{
Improving the accuracy of the magnetic field integral equation with the linear-linear basis functions
}

\author{
Özgür Ergül ${ }^{1}$ and Levent Gürel ${ }^{1}$
}

Received 29 June 2005; revised 24 January 2006; accepted 29 March 2006; published 18 July 2006.

[1] Basis functions with linear variations are investigated in terms of the accuracy of the magnetic field integral equation (MFIE) and the combined-field integral equation (CFIE), on the basis of recent reports indicating the inaccuracy of the MFIE. Electromagnetic scattering problems involving conducting targets with arbitrary geometries, closed surfaces, and planar triangulations are considered. Specifically, two functions with linear variations along the triangulation edges in both tangential and normal directions (linear normal and linear tangential (LN-LT) type) are defined. They are compared to the previously employed divergence-conforming Rao-Wilton-Glisson (RWG) and curl-conforming $\hat{\boldsymbol{n}} \times \mathrm{RWG}$ functions. Examples are presented to demonstrate the significant improvement in the accuracy of the MFIE and the CFIE gained by replacing the commonly used RWG functions with the LN-LT type functions.

Citation: Ergül, Ö., and L. Gürel (2006), Improving the accuracy of the magnetic field integral equation with the linear-linear basis functions, Radio Sci., 41, RS4004, doi:10.1029/2005RS003307.

\section{Introduction}

[2] For the solution of the three-dimensional (3-D) electromagnetic scattering problems involving perfectly conducting and closed surfaces with arbitrary shapes, the combined-field integral equation (CFIE) [Mautz and Harrington, 1978; Morita et al., 1990] is usually preferred over the electric field integral equation (EFIE) [Glisson and Wilton, 1980] and the magnetic field integral equation (MFIE) [Poggio and Miller, 1973; Zhang et al., 2003]. This is mainly because the CFIE is free of the internal resonance problem [Mautz and Harrington, 1978] and it generates better conditioned matrix equations [Gürel and Ergül, 2003] that are crucial for iterative solvers, such as the fast multipole method (FMM) [Coifman et al., 1993] and the multilevel fast multipole algorithm (MLFMA) [Lu and Chew, 1997; Chew et al., 2001], both based on the iterative solution of the integral equations discretized with the method of moments (MOM) [Harrington, 1968].

[3] For the discretization of 3-D arbitrary complicated surfaces, it has been most common and customary to use planar triangulations, on which the discretization of the

\footnotetext{
${ }^{1}$ Department of Electrical and Electronics Engineering, Bilkent University, Ankara, Turkey.

Copyright 2006 by the American Geophysical Union. 0048-6604/06/2005RS003307
}

induced unknown surface current is usually performed by using an expansion in a series of Rao-Wilton-Glisson (RWG) [Rao et al., 1982] functions. This approach is known to provide accurate results for the EFIE and therefore is also employed for the discretization and solution of the MFIE and CFIE [Hodges and RahmatSamii, 1997; Song et al., 1998; Shanker et al., 2000; Rius et al., 2001]. Unfortunately, compared to the EFIE results, the MFIE results are observed to be plagued with an inaccuracy problem persistent for a wide variety of scattering problems, even for the solution of simple geometries, such as the sphere [Ergül and Gürel, 2006].

[4] On the other hand, although the EFIE usually generates relatively more ill-conditioned systems and is prone to internal resonance problems, it also produces more accurate results, for example, radar cross section (RCS) values, than the MFIE. In other words, iterative solution of the EFIE is more difficult than the MFIE, however, the EFIE results are more accurate, if they can be obtained.

[5] In order to understand the fundamental reasons behind the inaccuracy of the MFIE, we investigated this problem in detail, especially by comparing the differences in the implementations of the EFIE and MFIE [Ergül and Gürel, 2004a]. Those investigations led to the clarification and improvement of some aspects of the MFIE, such as (1) improving the accuracy and efficiency of the computation of the singular MFIE integrals by using a novel singularity extraction scheme [Gürel and Ergül, 2005]; (2) extending the testing of the MFIE 
integrals from strictly inside the triangle to the edges of the triangle, similar to the practice in EFIE [Ergül and Gürel, 2005a]; and (3) clarifying, and thus avoiding the errors due to the incorrect uses of, the MFIE solid angle factor, which is not present in the EFIE [Ergül and Gürel, 2005b].

[6] Although each one of these improvements are important on their own, we were able to rule them out as the fundamental reason of the inaccuracy of the MFIE since the accuracy problem persevered even after employing all of the improvements. Consequently, further studies were stimulated for a better understanding of how the discretization of the MFIE, modeling of the surface current, and the choice of basis and testing functions affected the accuracy of the MFIE. Hence we have focused on the use of novel basis and testing functions for more accurate MFIE implementations [Ergül and Gürel, 2004b; Ergül and Gürel, 2006].

[7] Of the three possible formulations of the MFIE [Gürel and Ergül, 2005], the most common form has the differential operator on the Green's function so that the basis and testing functions are not restricted in terms of being divergence conforming or curl conforming. In other words, unlike the prevalent form of the EFIE, where the differential operators are placed on the basis and testing functions and thus restricting them to be divergence conforming, both divergence-conforming and curl-conforming functions can be used in the MFIE. In a recent study of the MFIE [Ergül and Gürel, 2006], we employed the curl-conforming $\hat{\boldsymbol{n}} \times$ RWG [Rao and Wilton, 1990; Sheng et al., 1998; Peterson, 2002] functions and obtained more accurate results compared to the divergence-conforming RWG functions. This finding encouraged us to further investigate the use of novel and higher-order basis functions in order to improve the accuracy of the MFIE to the level of the EFIE.

[8] In this paper, we compare the performances of linear basis functions defined on planar triangular domains in terms of the accuracy of the MFIE and CFIE. The testing functions are the same as the basis functions according to the Galerkin scheme. The functions under investigation are the divergence-conforming RWG and linear-linear (LL) [Trintinalia and Ling, 2001] functions, and the curl-conforming $\hat{\boldsymbol{n}} \times \mathrm{RWG}$ and $\hat{\boldsymbol{n}} \times \mathrm{LL}$ functions derived from them. As detailed in this paper, the LL and $\hat{\boldsymbol{n}} \times$ LL functions allow linear variation of the current along the edges of the triangulation at the cost of doubling the number of unknowns compared to the RWG and $\hat{\boldsymbol{n}} \times$ RWG functions. Nevertheless, the doubling of the number of unknowns due to the use of the LL and $\hat{\boldsymbol{n}} \times$ LL functions is abundantly worthwhile because of the significant improvement in the accuracy of the MFIE and CFIE. Examples of scattering problems and their results are presented in section 5 testifying to the improved accuracy obtained by using the LL and $\hat{\boldsymbol{n}} \times$ LL functions.

[9] Improving the accuracy of the MFIE by employing higher-order basis functions is also discussed in other contexts [Wang and Webb, 1997]. On a related note, higher-order interpolatory vector basis functions are presented for computational electromagnetics by Graglia et al. [1997]. In Appendix A, we present the LL functions as a decomposition of the RWG functions by following the notation by Graglia et al. [1997]. We note that $\hat{\boldsymbol{n}} \times$ LL functions can be obtained from the $\hat{\boldsymbol{n}} \times$ RWG functions by a similar decomposition.

\section{Linear Basis and Testing Functions}

[10] Table 1 lists the functions under investigation in this paper with some of their essential properties. The divergence-conforming RWG and the curl-conforming $\hat{\boldsymbol{n}} \times$ RWG functions were both employed for the MFIE previously [Hodges and Rahmat-Samii, 1997; Ergül and Gürel, 2006]. The divergence-conforming LL functions are suggested by Trintinalia and Ling [2001] in the context of the EFIE; however, in this work (and also the work by Ergül and Gürel [2004b]), we employ them in the MFIE and CFIE, in addition to the EFIE. The definition of the curl-conforming $\hat{\boldsymbol{n}} \times$ LL functions, which are derived from the LL functions in the same manner as the $\hat{\boldsymbol{n}} \times$ RWG functions are derived from the RWG functions, and their use in the MFIE are introduced for the first time in this paper.

[11] The RWG functions have the spatial distribution shown in Figure 1a, which can be written as

$$
\boldsymbol{f}^{R}(\boldsymbol{r})=\left\{\begin{aligned}
\frac{l}{2 A_{1}}\left(\boldsymbol{r}-\boldsymbol{r}_{1}\right), & r \in S_{1} \\
\frac{l}{2 A_{2}}\left(\boldsymbol{r}_{2}-\boldsymbol{r}\right), & r \in S_{2} \\
0, & \text { otherwise }
\end{aligned}\right.
$$

where $A_{1}$ and $A_{2}$ are the areas of the triangular surfaces $S_{1}$ and $S_{2}$, respectively, and $l$ is the length of the common edge shared by the triangles that we call the main edge. The surface divergence of the RWG functions is

$$
\nabla_{S} \cdot \boldsymbol{f}^{R}(\boldsymbol{r})=\left\{\begin{aligned}
\frac{l}{A_{1}}, & r \in S_{1} \\
\frac{-l}{A_{2}}, & r \in S_{2} \\
0, & \text { otherwise }
\end{aligned}\right.
$$

which is finite so that these functions are divergence conforming. In fact, the expression in (2) shows that the RWG functions support constant charge distribu- 
Table 1. Linear Basis and Testing Functions

\begin{tabular}{lccc}
\hline Function & Conformity & Type & Function/Edge \\
\hline RWG & Divergence & CN-LT & 1 \\
$\hat{\boldsymbol{n}} \times$ RWG & Curl & LN-CT & 1 \\
LL & Divergence & LN-LT & 2 \\
$\hat{\boldsymbol{n}} \times$ LL & Curl & LN-LT & 2 \\
\hline
\end{tabular}

tions on the triangular domains. It is common to classify these functions CN-LT type [Peterson et al., 1998], since their spatial distribution has constant normal $(\mathrm{CN})$ and linear tangential (LT) components at the main edge.

[12] The $\hat{\boldsymbol{n}} \times \mathrm{RWG}$ functions are derived from the RWG functions as

$$
\boldsymbol{f}^{n R}(\boldsymbol{r})=\hat{\boldsymbol{n}} \times \boldsymbol{f}^{R}(\boldsymbol{r}),
$$

where $\hat{\boldsymbol{n}}$ is the outwardly directed normal on the triangular domains, and they have the spatial distribution as shown in Figure 1b. These functions are the LN-CT (linear normal, constant tangential) type and curl conforming, that is, their surface curl

$$
\nabla_{S} \times \boldsymbol{f}^{n R}(\boldsymbol{r})=\hat{\boldsymbol{n}} \nabla_{S} \cdot \boldsymbol{f}^{R}(\boldsymbol{r})
$$

is finite.

[13] Besides the RWG and the $\hat{\boldsymbol{n}} \times$ RWG functions, we investigate the divergence-conforming LL functions, which are defined in pairs. As shown in Figure 2, there are two LL functions located at the same edge with the expressions for the LL functions of the second kind. The charge distribution implied by the expressions in (5) and (6) is

$$
\nabla_{S} \cdot \boldsymbol{f}^{L, 1}(\boldsymbol{r})=\nabla_{S} \cdot \boldsymbol{f}^{L, 2}(\boldsymbol{r})=\left\{\begin{array}{cc}
\frac{l}{2 A_{1}}, & r \in S_{1} \\
\frac{-l}{2 A_{2}}, & r \in S_{2} \\
0, & \text { otherwise, }
\end{array}\right.
$$

which is again constant and exactly half of that for the RWG functions in (2). Actually, this relation comes from the fact that

$$
\boldsymbol{f}^{R}(\boldsymbol{r})=\boldsymbol{f}^{L, 1}(\boldsymbol{r})+\boldsymbol{f}^{L, 2}(\boldsymbol{r})
$$

and the LL functions are simply the decomposition of the related RWG function as proved in Appendix A.

[14] Spatial distributions of the LL functions are depicted in Figure 2, where the edges of the triangles are labeled as $e_{p}, e_{z}$, and $e_{m}$ depending on the type of the LL function. Both kinds of the LL functions have the following properties:

[15] 1. They are parallel to one of the side edges $e_{p}$ over both of the triangles.

[16] 2. Their values change linearly along the edge $e_{p}$ and become maximum at the intersection of that edge with the main edge $e_{m}$. The value at this point is also the global maximum of the function.

[17] 3. Their values are exactly zero at the other side edge $e_{z}$.

[18] 4. They have continuous normal components across the main edge $e_{m}$.

$$
\boldsymbol{f}^{L, 1}(\boldsymbol{r})=\left\{\begin{aligned}
\frac{l}{4\left(A_{1}\right)^{2}}\left(\boldsymbol{r}-\boldsymbol{r}_{1}\right) \cdot\left[\left(\boldsymbol{r}_{4}-\boldsymbol{r}_{1}\right) \times \hat{\boldsymbol{n}}\right]\left(\boldsymbol{r}_{3}-\boldsymbol{r}_{1}\right), & r \in S_{1} \\
\frac{l}{4\left(A_{2}\right)^{2}}\left(\boldsymbol{r}-\boldsymbol{r}_{2}\right) \cdot\left[\left(\boldsymbol{r}_{4}-\boldsymbol{r}_{2}\right) \times \hat{\boldsymbol{n}}\right]\left(\boldsymbol{r}_{3}-\boldsymbol{r}_{2}\right), & r \in S_{2} \\
0, & \text { otherwise }
\end{aligned}\right.
$$

for the LL functions of the first kind, and

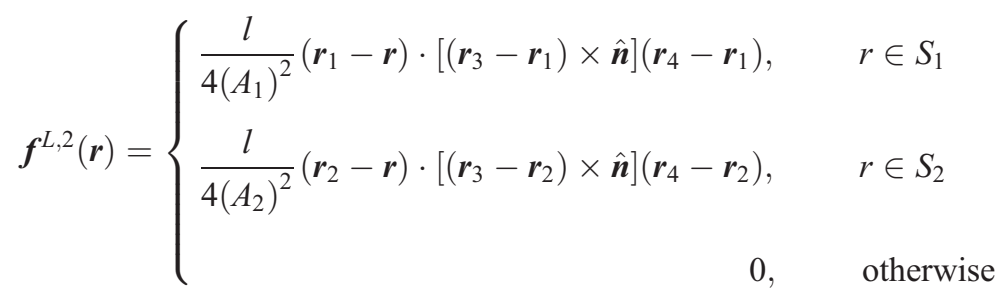




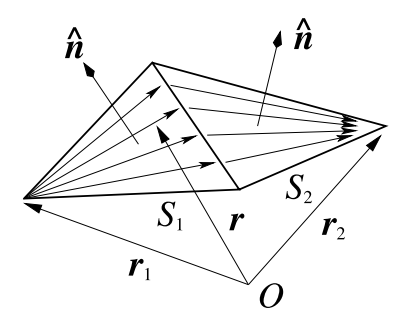

(a)

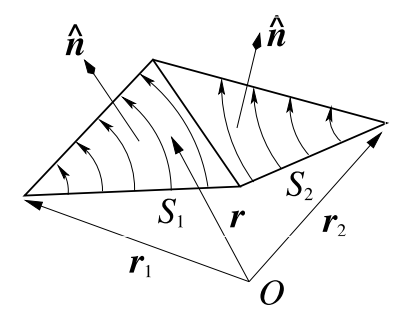

(b)

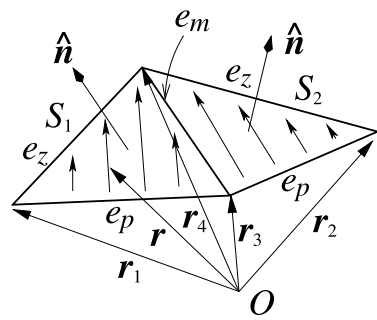

(a)

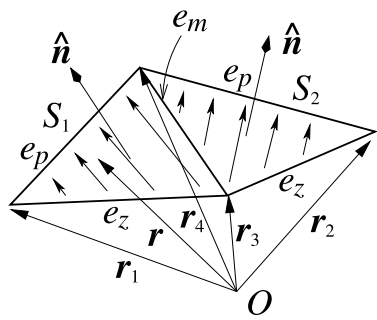

(b)

Figure 1. Spatial distributions of (a) RWG and (b) $\hat{\boldsymbol{n}} \times$ RWG functions.

Figure 3. Spatial distributions of (a) first-kind and (b) second-kind $\hat{\boldsymbol{n}} \times$ LL functions.

[19] 5. The variation at the main edge $e_{m}$ is linear in both perpendicular and tangential directions. As a result, the LL functions are classified as LN-LT type.

[20] Finally, the curl-conforming $\hat{\boldsymbol{n}} \times$ LL functions are derived from the LL functions as

$$
\begin{aligned}
\boldsymbol{f}^{n L, 1}(\boldsymbol{r}) & =\hat{\boldsymbol{n}} \times \boldsymbol{f}^{L, 1}(\boldsymbol{r}) \\
\boldsymbol{f}^{n L, 2}(\boldsymbol{r}) & =\hat{\boldsymbol{n}} \times \boldsymbol{f}^{L, 2}(\boldsymbol{r}),
\end{aligned}
$$

similar to the derivation of $\hat{\boldsymbol{n}} \times$ RWG functions in (3), and we note that

$$
\begin{aligned}
& \nabla_{S} \times \boldsymbol{f}^{n L, 1}(\boldsymbol{r})=\hat{\boldsymbol{n}} \nabla_{S} \cdot \boldsymbol{f}^{L, 1}(\boldsymbol{r}) \\
& \nabla_{S} \times \boldsymbol{f}^{n L, 2}(\boldsymbol{r})=\hat{\boldsymbol{n}} \nabla_{S} \cdot \boldsymbol{f}^{L, 2}(\boldsymbol{r}) .
\end{aligned}
$$

Similar to the LL functions, the $\hat{\boldsymbol{n}} \times$ LL functions are also defined in pairs located at the same edge with spatial distributions pictured in Figure 3. Spatial distributions of the $\hat{\boldsymbol{n}} \times$ LL functions have the following properties:

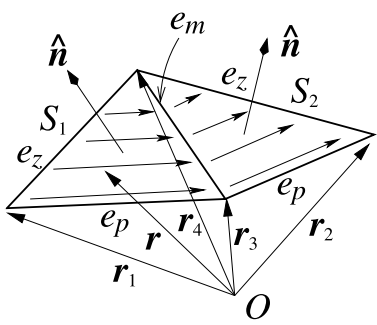

(a)

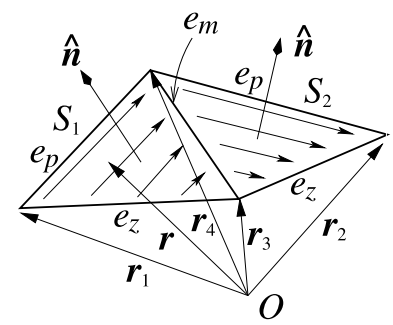

(b)

Figure 2. Spatial distributions of (a) first-kind and (b) second-kind LL functions.

[21] 1. They are perpendicular to one of the side edges $e_{p}$ over both triangles.

[22] 2. Similar to the LL functions, their values at the edge $e_{p}$ change linearly becoming maximum at the intersection with the main edge $e_{m}$.

[23] 3. Their values are again zero at the other side edge $e_{z}$.

[24] 4. They have continuous tangential components across the main edge $e_{m}$.

[25] 5. Similar to the LL functions, $\hat{\boldsymbol{n}} \times$ LL functions are also LN-LT type and they have spatial distributions with linear variation in both tangential and normal directions at the main edge $e_{m}$.

[26] What makes the LL and $\hat{\boldsymbol{n}} \times$ LL functions good candidates for the MFIE and CFIE is that these functions are both LN-LT type. Our studies of the accuracy of the MFIE indicated that a proper modeling of the current is crucial, especially along the physical edges of the discretized geometry [Ergül and Gürel, 2006]. Therefore this work aims to demonstrate whether the additional degrees of freedom (DOFs) present in the LL and $\hat{\boldsymbol{n}} \times$ LL functions (compared to the RWG and $\hat{\boldsymbol{n}} \times \mathrm{RWG}$ functions) will improve the accuracy of the MFIE, and consequently, the CFIE.

\section{MOM Formulations}

[27] For conducting scatterers with closed surfaces, the MFIE can be written by using the boundary condition for the tangential magnetic field on the surface as

$$
\boldsymbol{J}(\boldsymbol{r})-\hat{\boldsymbol{n}} \times \int_{S} d \boldsymbol{r}^{\prime} \boldsymbol{J}\left(\boldsymbol{r}^{\prime}\right) \times \nabla^{\prime} g\left(\boldsymbol{r}, \boldsymbol{r}^{\prime}\right)=\hat{\boldsymbol{n}} \times \boldsymbol{H}^{i}(\boldsymbol{r}),
$$

where the scattered magnetic field is expressed in terms of the induced (unknown) surface current $\boldsymbol{J}(\boldsymbol{r})$. In (11), the observation point $\boldsymbol{r}$ approaches the surface from the 


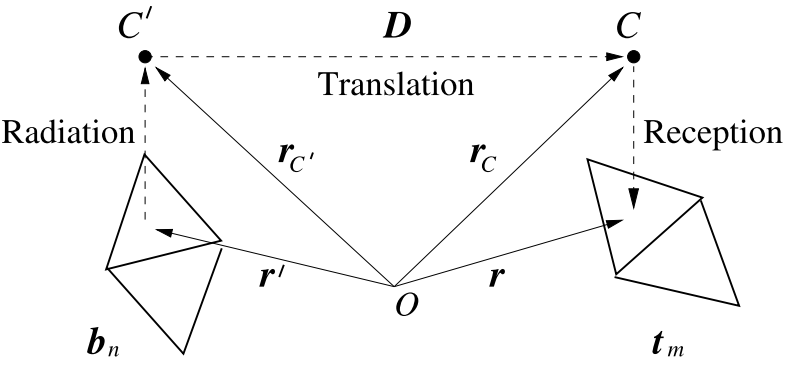

Figure 4. Calculation of the interactions in the FMM.

outside, $\hat{\boldsymbol{n}}$ is the outwardly directed normal, $\boldsymbol{H}^{i}(\boldsymbol{r})$ is the incident magnetic field, and

$$
g\left(\boldsymbol{r}, \boldsymbol{r}^{\prime}\right)=\frac{e^{i k R}}{4 \pi R} \quad\left(R=\left|\boldsymbol{r}-\boldsymbol{r}^{\prime}\right|\right)
$$

denotes the free-space Green's function in phasor notation with the $e^{-i w t}$ convention.

[28] With the application of the MOM discretization to the MFIE in (11), an $N \times N$ matrix equation

$$
\sum_{n=1}^{N} Z_{m n} a_{n}=v_{m}, \quad m=1, \ldots, N
$$

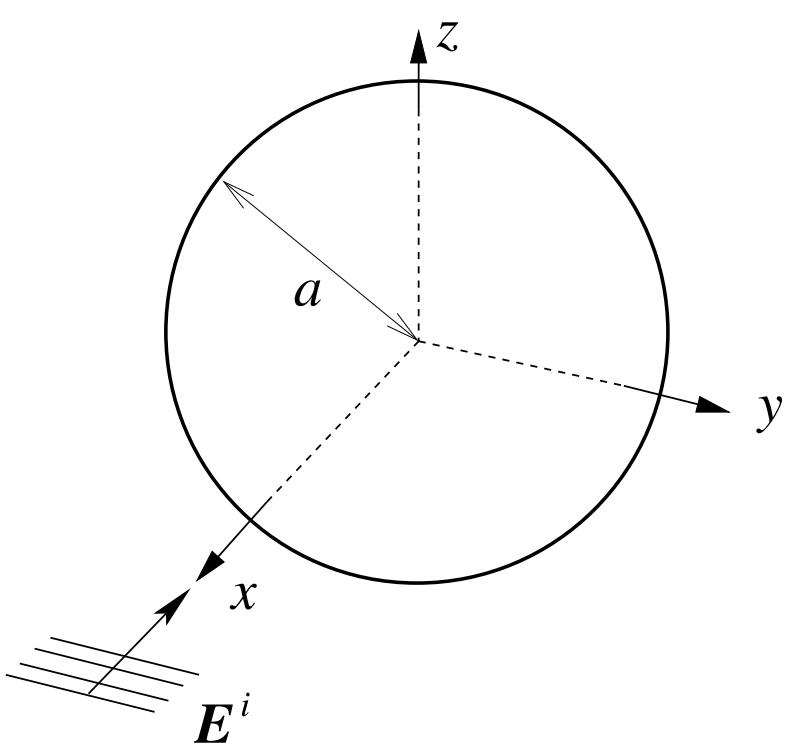

Figure 5. Perfectly conducting sphere of radius $a$, illuminated by a plane wave propagating in the $-x$ direction with the electric field polarization in the $y$ direction.

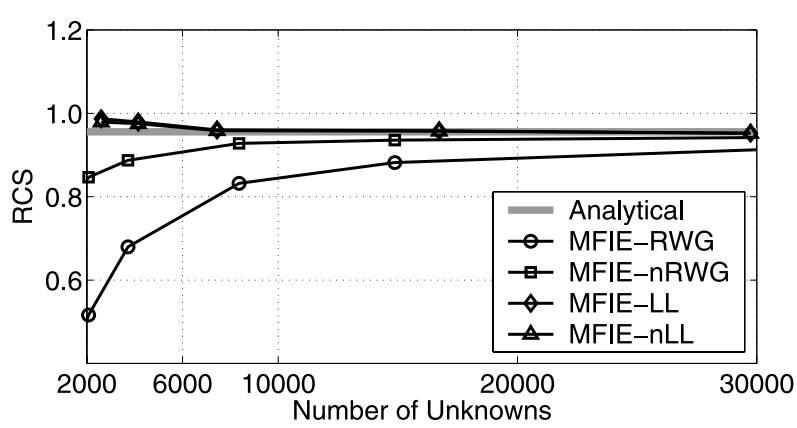

(a)

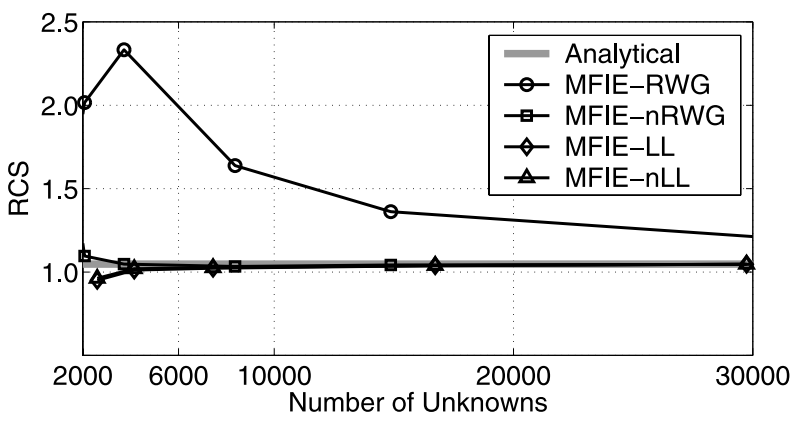

(b)

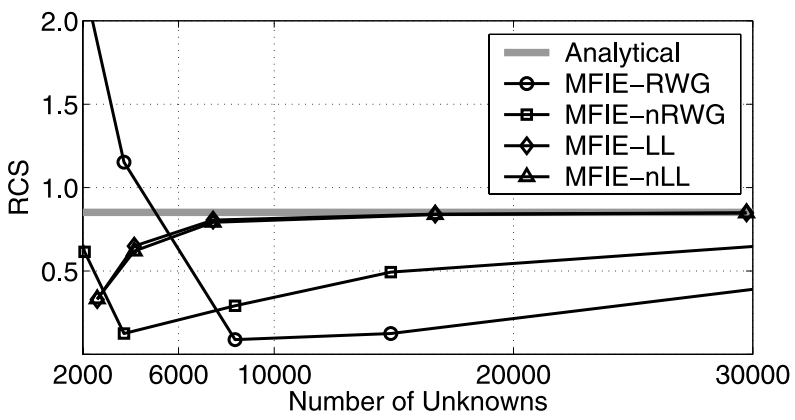

(c)

Figure 6. Normalized backscattered $\operatorname{RCS}\left(\mathrm{RCS} / \pi a^{2}\right.$, dimensionless) of the perfectly conducting sphere (in Figure 5) of radius (a) $a=\lambda$, (b) $a=1.5 \lambda$, and (c) $a=2 \lambda$ with respect to the number of unknowns.

is obtained, where $Z_{m n}$ is the matrix element derived as

$$
\begin{aligned}
Z_{m n}^{M}= & \int_{S_{m}} d \boldsymbol{r} \boldsymbol{t}_{m}(\boldsymbol{r}) \cdot \boldsymbol{b}_{n}(\boldsymbol{r}) \\
& -\int_{S_{m}} d \boldsymbol{r} \boldsymbol{t}_{m}(\boldsymbol{r}) \cdot \hat{\boldsymbol{n}} \times \int_{S_{n}} d \boldsymbol{r}^{\prime} \boldsymbol{b}_{n}\left(\boldsymbol{r}^{\prime}\right) \times \nabla^{\prime} g\left(\boldsymbol{r}, \boldsymbol{r}^{\prime}\right)
\end{aligned}
$$




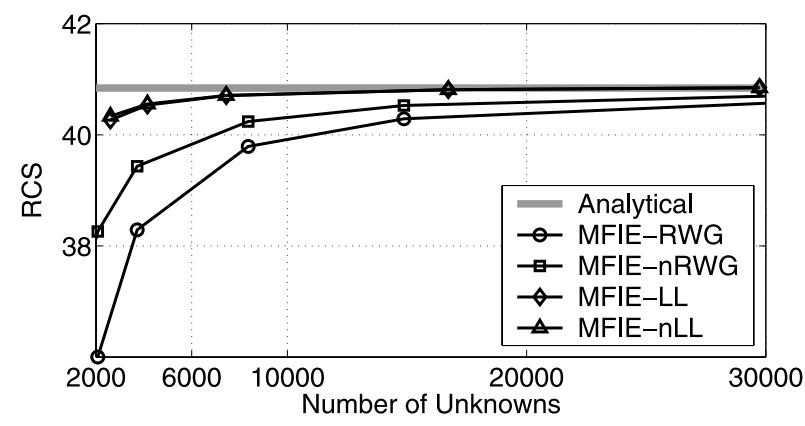

(a)

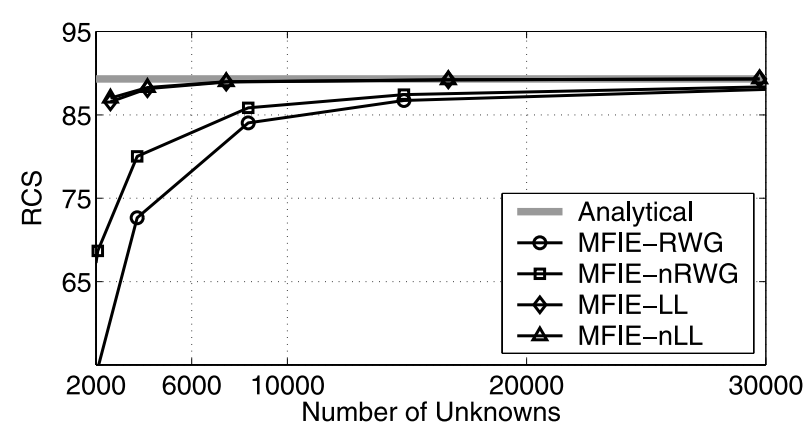

(b)

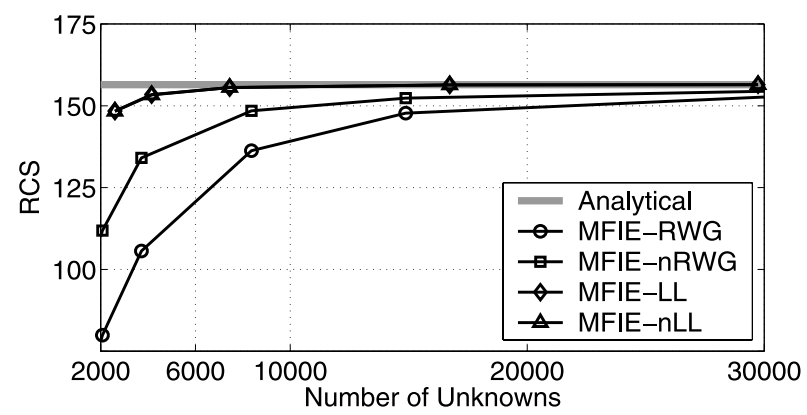

(c)

Figure 7. Normalized forward-scattered RCS (RCS/ $\pi a^{2}$, dimensionless) of the perfectly conducting sphere (in Figure 5) of radius (a) $a=\lambda$, (b) $a=1.5 \lambda$, and (c) $a=$ $2 \lambda$ with respect to the number of unknowns.

and $v_{m}$ is the element of the excitation vector derived as

$$
v_{m}=\int_{S_{m}} d \boldsymbol{r} \boldsymbol{t}_{m}(\boldsymbol{r}) \cdot \hat{\boldsymbol{n}} \times \boldsymbol{H}^{i}(\boldsymbol{r}) .
$$

In (14) and (15), $S_{n}$ and $S_{m}$ represent the surfaces, on which the $n$th basis function $\boldsymbol{b}_{n}(\boldsymbol{r})$ and the $m$ th testing function $\boldsymbol{t}_{m}(\boldsymbol{r})$ are defined, respectively.
[29] For the divergence-conforming RWG and LL functions, we use the expression in (14) in order to evaluate the matrix elements. Although the same expression is also valid for the curl-conforming $\hat{\boldsymbol{n}} \times \mathrm{RWG}$ and $\hat{\boldsymbol{n}} \times$ LL functions, we prefer the modified expression for these functions as

$$
\begin{aligned}
Z_{m n}^{M}= & \int_{S_{m}} d \boldsymbol{r} \boldsymbol{t}_{m}(\boldsymbol{r}) \cdot \boldsymbol{b}_{n}(\boldsymbol{r}) \\
& +\int_{S_{m}} d \boldsymbol{r} \boldsymbol{t}_{m}(\boldsymbol{r}) \cdot \hat{\boldsymbol{n}} \times \int_{S_{n}} d \boldsymbol{r}^{\prime} \nabla^{\prime} \times\left[\boldsymbol{b}_{n}\left(\boldsymbol{r}^{\prime}\right) g\left(\boldsymbol{r}, \boldsymbol{r}^{\prime}\right)\right] \\
& -\int_{S_{m}} d \boldsymbol{r t _ { m }}(\boldsymbol{r}) \cdot \hat{\boldsymbol{n}} \times \int_{S_{n}} d \boldsymbol{r}^{\prime} g\left(\boldsymbol{r}, \boldsymbol{r}^{\prime}\right) \nabla^{\prime} \times \boldsymbol{b}_{n}\left(\boldsymbol{r}^{\prime}\right)
\end{aligned}
$$

for efficiency. In (16), the inner integral of the second term can be manipulated into a line integral over the edges around the basis function [Ergül and Gürel, 2006]. Moreover, the use of the expressions either in (4) or (10), for the $\hat{\boldsymbol{n}} \times \mathrm{RWG}$ and $\hat{\boldsymbol{n}} \times$ LL functions, respectively, simplifies the double integral in the third term of (16) compared to the double-integral term in (14).

[30] Evaluation of the integrals in (14) and (16) has been extensively investigated in various references [Hodges and Rahmat-Samii, 1997; Ylä-Oijala and Taskinen, 2003; Ergül and Gürel, 2006]. We will not repeat the details here, but will simply summarize the steps for an efficient and reliable implementation:

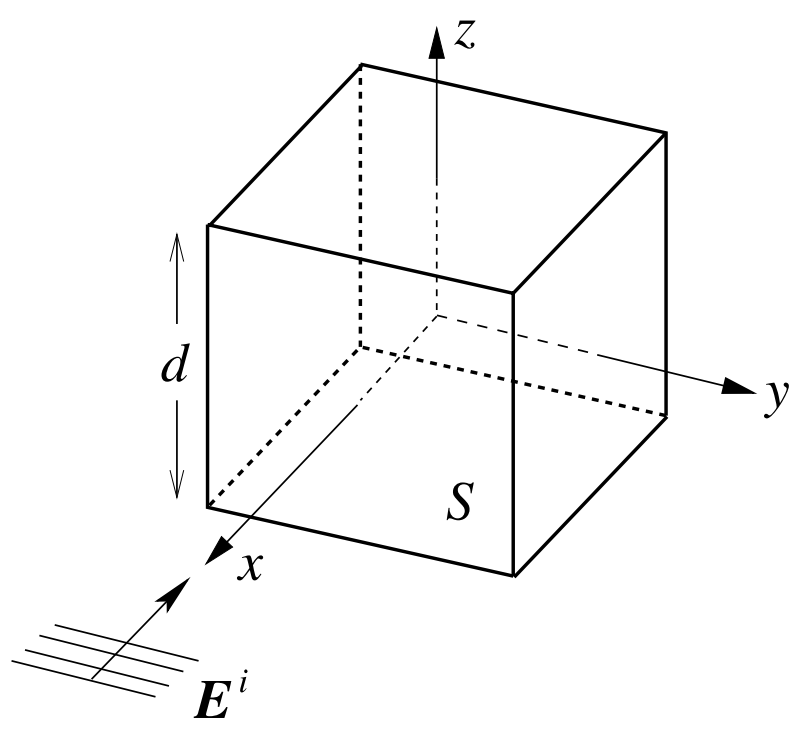

Figure 8. Perfectly conducting cube with edges of $d=$ $1 \mathrm{~m}$, illuminated by a plane wave propagating in the $-x$ direction with the electric field polarization in the $y$ direction. 


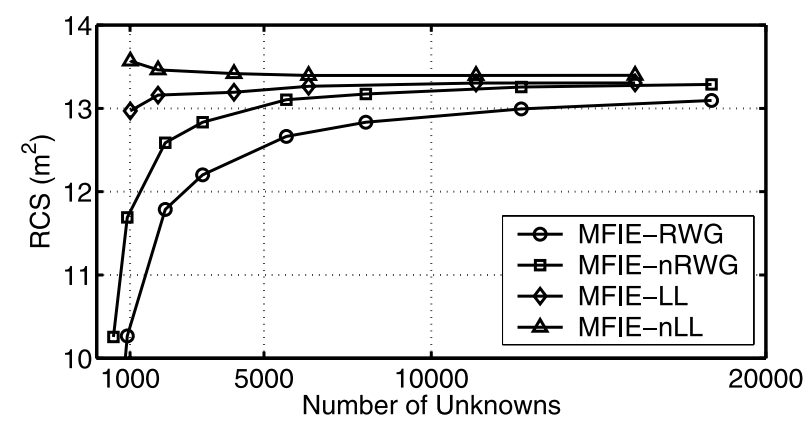

(a)

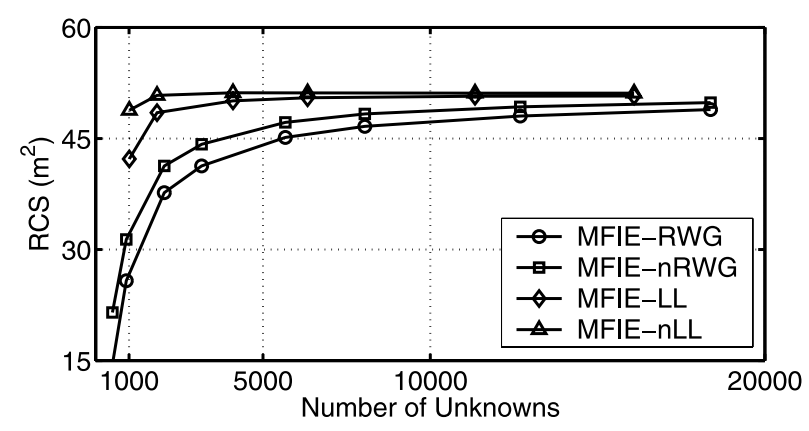

(b)

Figure 9. Backscattered RCS $\left(\mathrm{m}^{2}\right)$ of the perfectly conducting cube in Figure 8 with (a) $d=\lambda$ and (b) $d=2 \lambda$ with respect to the number of unknowns.

[31] 1. The loops are constructed over the triangles instead of the functions. In such a construction, the integrals over the basis and testing functions must be divided into many basic (double) integrals that are independent from the alignment of the functions.

[32] 2. The basic integrals are evaluated in two steps; first, the inner integrals are calculated, and then, they are used in forming the integrands of the outer integrals.

[33] 3. The inner integrals are commonly shared by more than one basic integral. The inner integrals are performed via a decomposition into numerical and analytical parts [Graglia, 1993]. The singularities arising from the Green's function are extracted this way in order to avoid numerical difficulties and inaccuracies.

[34] 4. The MFIE includes singularities also in the outer integrals related to the interactions between neighboring functions. A singularity extraction method to handle those singularities is useful to improve both efficiency and accuracy [Gürel and Ergül, 2005].

[35] 5. Numerical integrations are performed by using adaptive methods employing low-order Gaussian quadratures. Such adaptive methods achieve the preset re- quired error bounds by sampling the integration points efficiently.

[36] In the construction of the CFIE matrix via

$$
Z_{m n}^{C}=\alpha Z_{m n}^{E}+\frac{i}{k}(1-\alpha) Z_{m n}^{M},
$$

the contribution of the EFIE is derived as

$$
\begin{aligned}
Z_{m n}^{E}= & \int_{S_{m}} d \boldsymbol{r} t_{m}(\boldsymbol{r}) \cdot \int_{S_{n}} d \boldsymbol{r}^{\prime} g\left(\boldsymbol{r}, \boldsymbol{r}^{\prime}\right) \boldsymbol{b}_{n}\left(\boldsymbol{r}^{\prime}\right) \\
& +\frac{1}{k^{2}} \int_{S_{m}} d \boldsymbol{r} \boldsymbol{r}_{m}(\boldsymbol{r}) \cdot \nabla \int_{S_{n}} d \boldsymbol{r}^{\prime} g\left(\boldsymbol{r}, \boldsymbol{r}^{\prime}\right) \nabla^{\prime} \cdot \boldsymbol{b}_{n}\left(\boldsymbol{r}^{\prime}\right) .
\end{aligned}
$$

The second term of (18), which is hypersingular, is usually manipulated as

$$
\begin{aligned}
Z_{m n}^{E}= & \int_{S_{m}} d \boldsymbol{r} \boldsymbol{t}_{m}(\boldsymbol{r}) \cdot \int_{S_{n}} d \boldsymbol{r}^{\prime} g\left(\boldsymbol{r}, \boldsymbol{r}^{\prime}\right) \boldsymbol{b}_{n}\left(\boldsymbol{r}^{\prime}\right) \\
& -\frac{1}{k^{2}} \int_{S_{m}} d \boldsymbol{r}\left[\nabla \cdot \boldsymbol{t}_{m}(\boldsymbol{r})\right] \int_{S_{n}} d \boldsymbol{r}^{\prime} g\left(\boldsymbol{r}, \boldsymbol{r}^{\prime}\right)\left[\nabla^{\prime} \cdot \boldsymbol{b}_{\boldsymbol{n}}\left(\boldsymbol{r}^{\prime}\right)\right],
\end{aligned}
$$

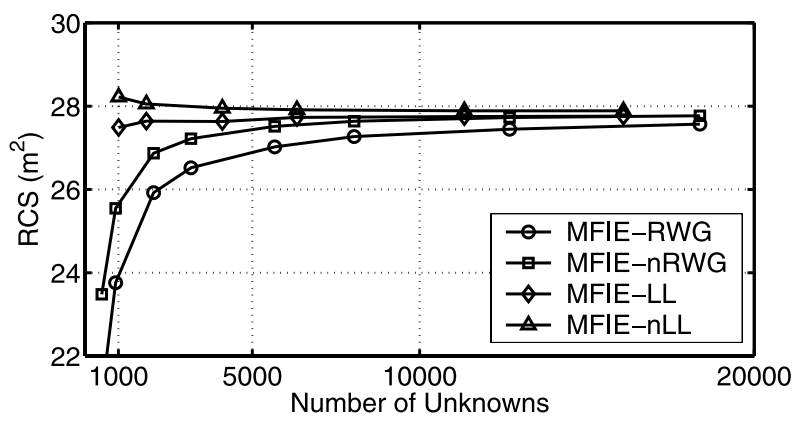

(a)

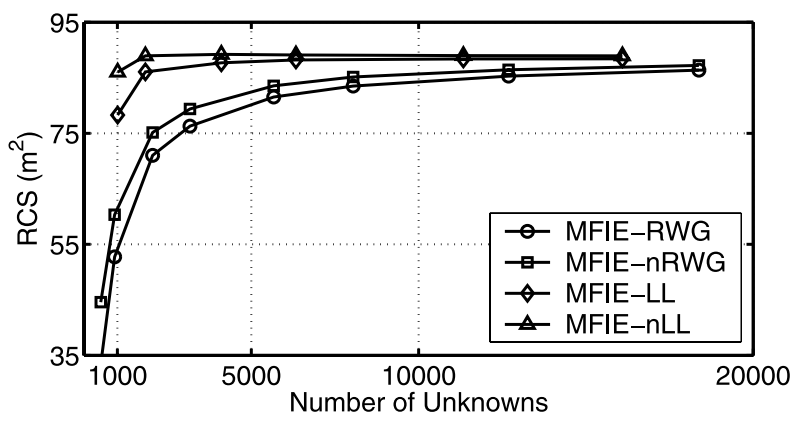

(b)

Figure 10. Forward-scattered RCS $\left(\mathrm{m}^{2}\right)$ of the perfectly conducting cube in Figure 8 with (a) $d=\lambda$ and (b) $d=2 \lambda$ with respect to the number of unknowns. 


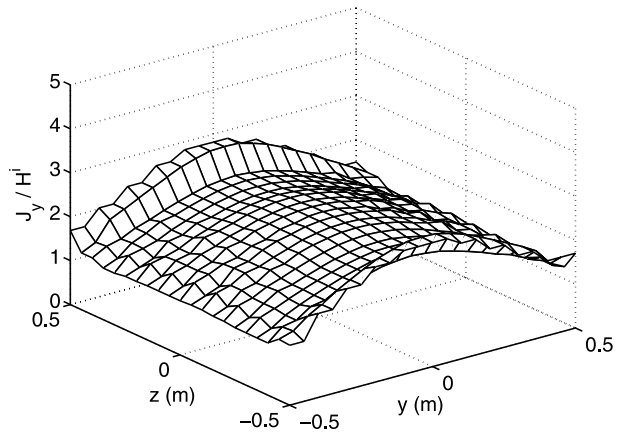

(a)

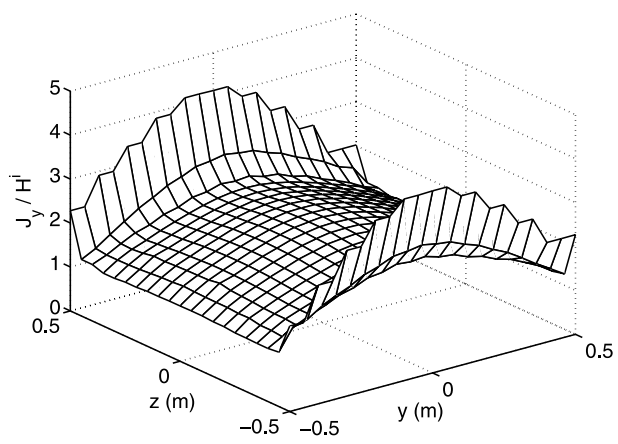

(c)

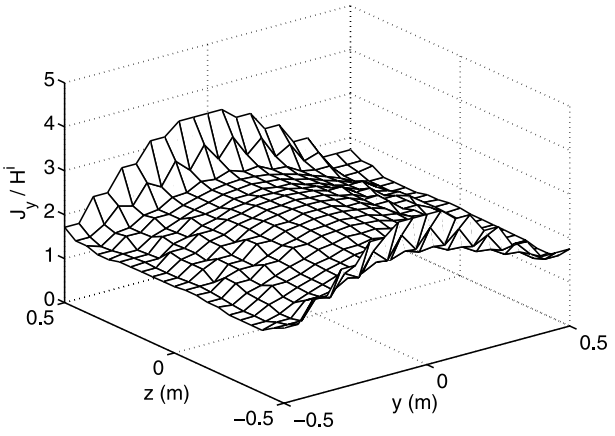

(b)

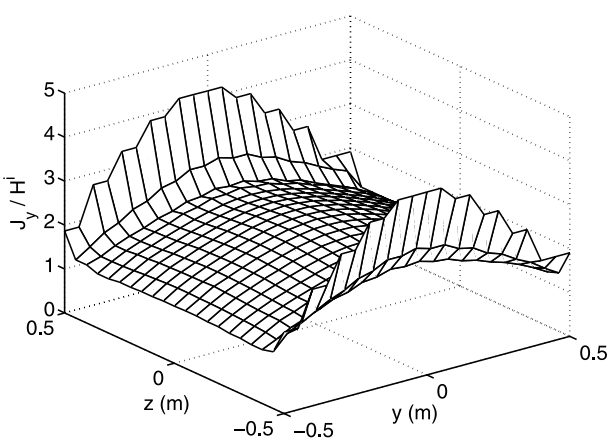

(d)

Figure 11. Magnitude of the $y$ component of the normalized surface current $\left|J_{y} / H^{i}\right|$ induced on the front surface (at $x=d / 2$ ) of the perfectly conducting cube in Figure 8 with $d=\lambda$ and triangulation size of about $\lambda / 10$, modeled by (a) RWG, (b) $\hat{\boldsymbol{n}} \times \mathrm{RWG}$, (c) LL, and (d) $\hat{\boldsymbol{n}} \times$ LL functions. The total number of unknowns of the problem is 2052 for the RWG and $\hat{\boldsymbol{n}} \times$ RWG functions and 4104 for the LL and $\hat{\boldsymbol{n}} \times \mathrm{LL}$ functions.

which restricts the basis and testing functions to be divergence conforming. In this work, we follow the convention and employ only the divergence-conforming RWG and LL functions in the EFIE and CFIE.

\section{FMM and MLFMA Formulations}

[37] As the problem size gets larger and the number of unknowns increases, the MOM becomes insufficient to solve the scattering problem and we employ fast iterative methods such as the FMM and the MLFMA. In these methods, only the near-field interactions are calculated directly as in (14), (16), and (19). The rest of the interactions are calculated in a faster way on the basis of the factorization of the Green's function. In the FMM, the matrix elements related to the far-field interactions are derived as [Chew et al., 2001]

$$
Z_{m n}^{M}=\frac{k^{2}}{(4 \pi)^{2}} \int d^{2} \hat{\boldsymbol{k}} \boldsymbol{F}_{C m}^{M}(\hat{\boldsymbol{k}}) T_{L}(k,|\boldsymbol{D}|, \hat{\boldsymbol{D}} \cdot \hat{\boldsymbol{k}}) \cdot \boldsymbol{F}_{C^{\prime} n}^{M}(\hat{\boldsymbol{k}})
$$

for the MFIE and

$$
Z_{m n}^{E}=\frac{i k}{(4 \pi)^{2}} \int d^{2} \hat{\boldsymbol{k}}_{C m}^{E}(\hat{\boldsymbol{k}}) T_{L}(k,|\boldsymbol{D}|, \hat{\boldsymbol{D}} \cdot \hat{\boldsymbol{k}}) \cdot \boldsymbol{F}_{C^{\prime} n}^{E}(\hat{\boldsymbol{k}})
$$

for the EFIE, where

$$
T_{L}(k,|\boldsymbol{D}|, \hat{\boldsymbol{D}} \cdot \hat{\boldsymbol{k}})=\sum_{l=0}^{L} i^{l}(2 l+1) h_{l}^{(1)}(k D) P_{l}(\hat{\boldsymbol{D}} \cdot \hat{\boldsymbol{k}})
$$

is the translation function written in terms of the spherical Hankel function of the first kind $h_{l}^{(1)}$ and Legendre polynomial $P_{l}$. For the CFIE, (20) and (21) are combined as stated in (17).

[38] The evaluation of (20) and (21) is illustrated in Figure 4. First, the two kinds of radiation of the $n$th basis function, namely, $\boldsymbol{F}_{C^{\prime} n}^{M}(\hat{k})$ for the MFIE and $\boldsymbol{F}_{C^{\prime} n}^{E}(\hat{k})$ for the EFIE, are calculated with respect to a near point 


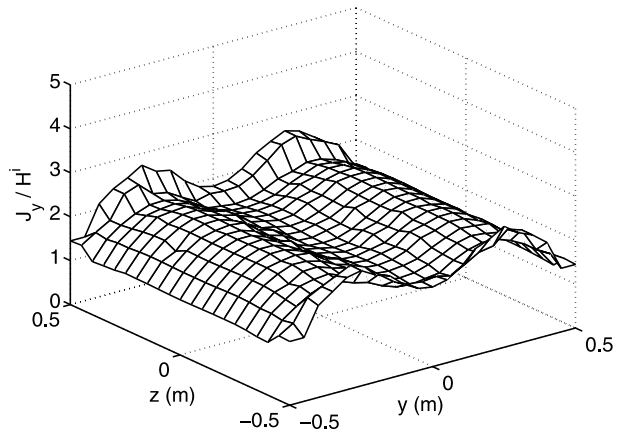

(a)

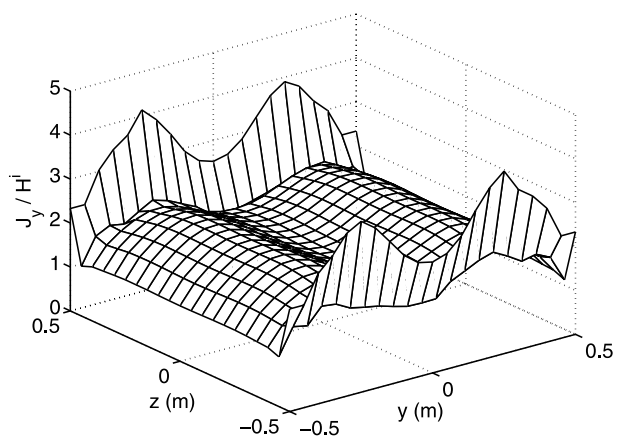

(c)

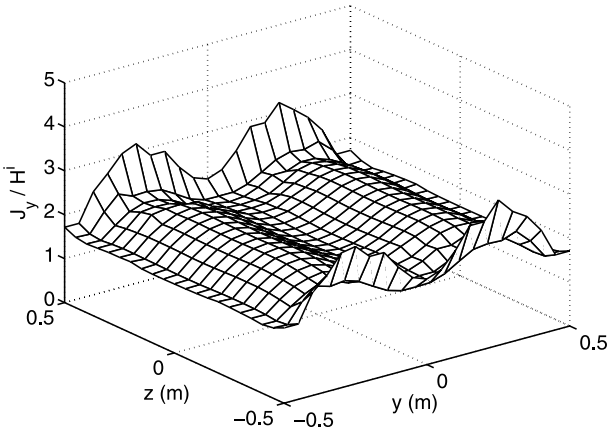

(b)

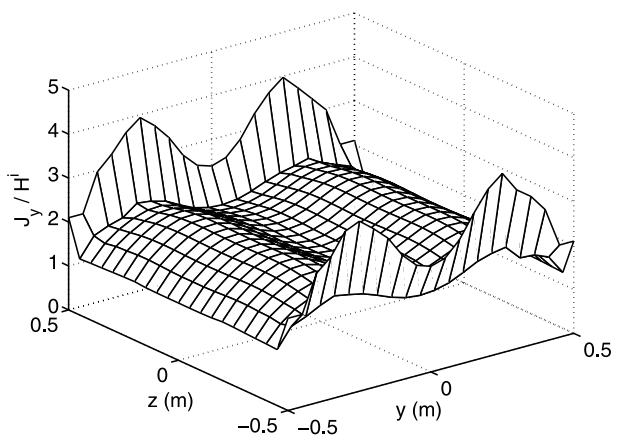

(d)

Figure 12. Magnitude of the $y$ component of the normalized surface current $\left|J_{y} / H^{i}\right|$ induced on the front surface (at $x=d / 2$ ) of the perfectly conducting cube in Figure 8 with $d=2 \lambda$ and triangulation size of about $\lambda / 10$, modeled by (a) RWG, (b) $\hat{\boldsymbol{n}} \times \mathrm{RWG}$, (c) LL, and (d) $\hat{\boldsymbol{n}} \times$ LL functions. The total number of unknowns of the problem is 8046 for the RWG and $\hat{\boldsymbol{n}} \times \mathrm{RWG}$ functions and 16,092 for the LL and $\hat{\boldsymbol{n}} \times$ LL functions.

$C^{\prime}$ located at $\boldsymbol{r}_{C^{\prime}}$. Then, the radiation is translated by $T_{L}(k$, $|\boldsymbol{D}|, \hat{D} \cdot \hat{k})$ into an incoming wave at point $C$ located at $\boldsymbol{r}_{C}=\boldsymbol{r}_{C^{\prime}}+\boldsymbol{D}$. Finally, the incoming wave is received by the $m$ th testing function that has the receiving patterns $\boldsymbol{F}_{C m}^{M}(\hat{k})$ for the MFIE and $\boldsymbol{F}_{C m}^{E}(\hat{k})$ for the EFIE with respect to a close point $C$.

[39] The FMM interactions related to different types of functions have the common expression for the translation function in (22), but the radiation and receiving patterns are specific to the type of the function. These patterns are derived as [Chew et al., 2001]

$$
\boldsymbol{F}_{C^{\prime} n}^{M}(\hat{\boldsymbol{k}})=\int_{S_{n}} d \boldsymbol{r}^{\prime} e^{-i \boldsymbol{k} \cdot\left(\boldsymbol{r}^{\prime}-\boldsymbol{r}_{C^{\prime}}\right)} \boldsymbol{b}_{n}\left(\boldsymbol{r}^{\prime}\right)
$$

and

$$
\boldsymbol{F}_{C m}^{M}(\hat{\boldsymbol{k}})=-\hat{\boldsymbol{k}} \times \int_{S_{m}} d \boldsymbol{r} e^{i \boldsymbol{k} \cdot\left(\boldsymbol{r}-\boldsymbol{r}_{C}\right)} \boldsymbol{t}_{m}(\boldsymbol{r}) \times \hat{\boldsymbol{n}}
$$

for the MFIE, and

$$
\boldsymbol{F}_{C^{\prime} n}^{E}(\hat{\boldsymbol{k}})=\int_{S_{n}} d \boldsymbol{r}^{\prime} e^{-i \boldsymbol{k} \cdot\left(\boldsymbol{r}^{\prime}-\boldsymbol{r}_{C^{\prime}}\right)}(\boldsymbol{I}-\hat{\boldsymbol{k}} \hat{\boldsymbol{k}}) \cdot \boldsymbol{b}_{n}\left(\boldsymbol{r}^{\prime}\right)
$$

and

$$
\boldsymbol{F}_{C m}^{E}(\hat{\boldsymbol{k}})=\int_{S_{m}} d \boldsymbol{r} e^{i \boldsymbol{k} \cdot\left(\boldsymbol{r}-\boldsymbol{r}_{C}\right)}(\boldsymbol{I}-\hat{\boldsymbol{k}} \hat{\boldsymbol{k}}) \cdot \boldsymbol{t}_{m}(\boldsymbol{r})
$$

for the EFIE. Using linear functions, the evaluation of the integrals in (23)-(26) can be performed analytically. In CFIE implementations, only the expressions in (24) and (25) require calculation, since (26) is simply the complex conjugate of (25) for $m=n$ provided that the Galerkin approach is used; and (23) can be reduced to (25) by discarding the radial component of the radiation that is expected to be canceled because of the dot product in (20). 


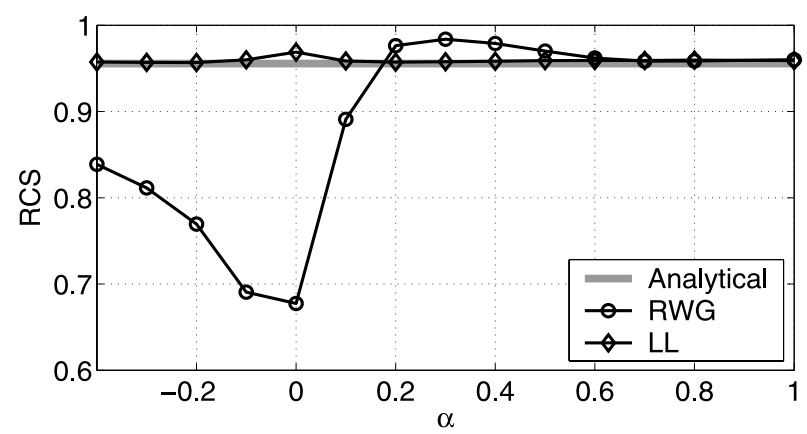

(a)

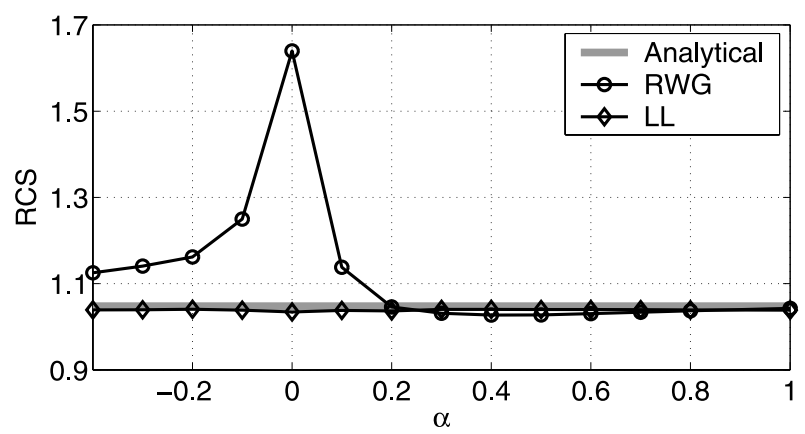

(b)

Figure 13. Normalized backscattered RCS $\left(\mathrm{RCS} / \pi a^{2}\right.$, dimensionless) of the perfectly conducting sphere (in Figure 5) of radius (a) $a=\lambda$ and (b) $a=1.5 \lambda$ with respect to $\alpha$ in the CFIE. The number of unknowns is 3723 for RWG and 7446 for LL (Figure 13a) and 8364 for RWG and 16,728 for LL (Figure 13b).

[40] Using the FMM, the matrix-vector multiplication required by the iterative algorithms is performed with $O\left(N^{3 / 2}\right)$ floating-point operations using $O\left(N^{3 / 2}\right)$ memory. Both of these complexities are reduced to $O(N \log N)$ by the MLFMA, where the FMM is applied in a structure of levels formed by the concept of "grouping the groups." The multilevel algorithm is detailed by Chew et al. [2001].

\section{Results}

[41] To compare the basis (and testing) functions in terms of how they affect the accuracy of the MFIE implementations, we first present the results of a scattering problem involving a perfectly conducting sphere of radius $a$ as shown in Figure 5. The sphere is illuminated by a plane wave propagating in the $-x$ direction with the electric field polarization in the $y$ direction. The problem is solved with the FMM or MLFMA implementations employing the RWG, $\hat{\boldsymbol{n}} \times \mathrm{RWG}, \mathrm{LL}$, and $\hat{\boldsymbol{n}} \times \mathrm{LL}$ functions. Figures 6 and 7 display the normalized backscattered and forward-scattered radar cross section $\left(\mathrm{RCS} / \pi a^{2}\right)$ values, respectively, for various sizes of the sphere when $a=\lambda, 1.5 \lambda$, and $2 \lambda$. For those sizes of the sphere, $\lambda / 10$ meshing leads to triangulations with 3723 , 8364 , and 14,871 edges, respectively.

[42] In Figures 6 and 7, the RCS values are plotted with respect to the number of unknowns, which corresponds to the number of edges for the RWG and $\hat{\boldsymbol{n}} \times$ RWG functions, but twice that for the LL and $\hat{\boldsymbol{n}} \times \mathrm{LL}$ functions. To compare the performances of the functions, convergence to the analytically calculated value is investigated. It can be observed that the convergence is significantly faster for the LN-LT type functions, namely, the divergence-conforming LL and the curl-conforming $\hat{\boldsymbol{n}} \times$ LL functions. In other words, for a given number of unknowns, MFIE solutions obtained by these functions are more accurate than those obtained by the RWG and $\hat{\boldsymbol{n}} \times$ RWG functions. We also note that the $\hat{\boldsymbol{n}} \times \mathrm{RWG}$ functions give more accurate RCS values compared to

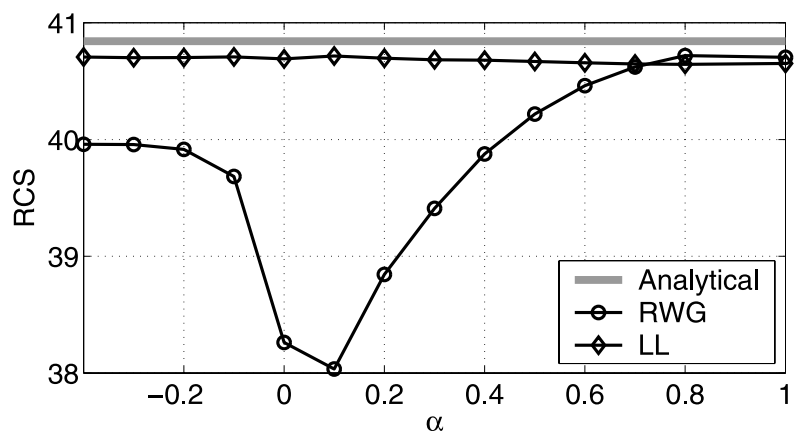

(a)

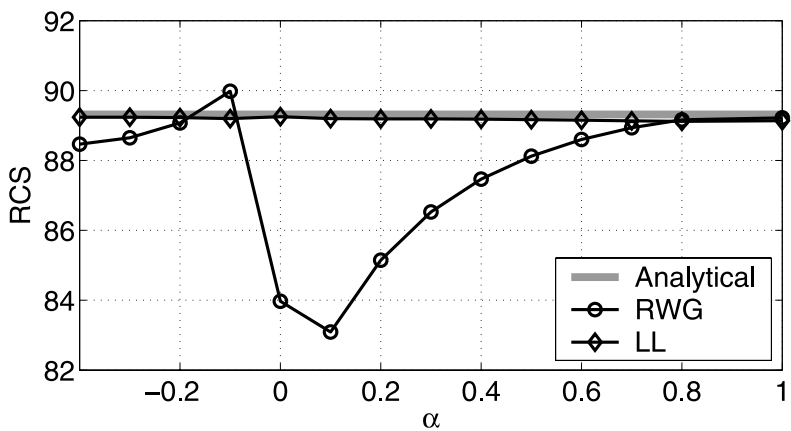

(b)

Figure 14. Normalized forward-scattered RCS (RCS/ $\pi a^{2}$, dimensionless) of the perfectly conducting sphere (in Figure 5) of radius (a) $a=\lambda$ and (b) $a=1.5 \lambda$ with respect to $\alpha$ in the CFIE. The number of unknowns is 3723 for RWG and 7446 for LL (Figure 14a) and 8364 for RWG and 16,728 for LL (Figure 14b). 


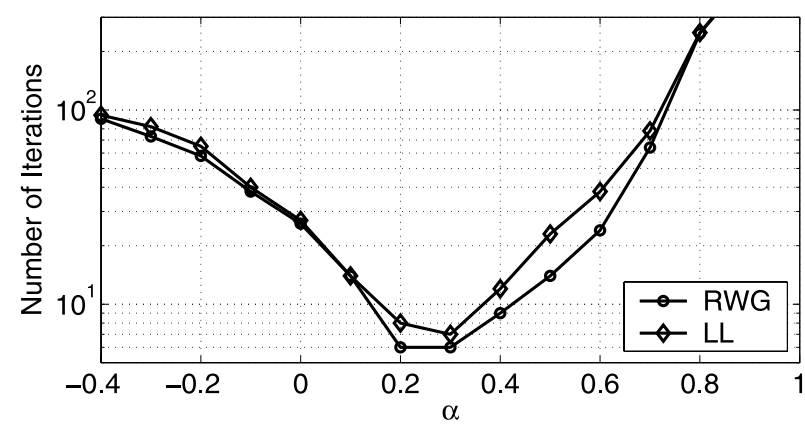

(a)

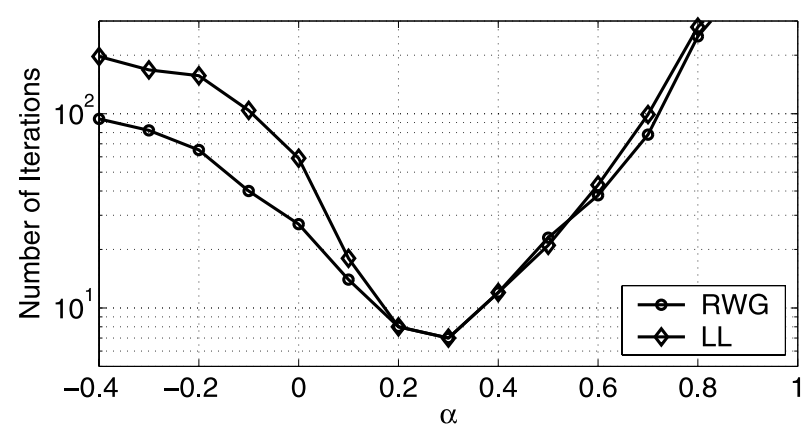

(b)

Figure 15. Iteration counts required to reach $10^{-3}$ residual error in the solution of the scattering problem of the sphere (in Figure 5) of radius (a) $a=\lambda$ (3723 edges) and (b) $a=1.5 \lambda$ (8364 edges) with respect to $\alpha$ in CFIE.

the RWG functions [Ergül and Gürel, 2006], but the accuracy is relatively poor compared to the LL and $\hat{\boldsymbol{n}} \times$ LL functions.

[43] The RCS values in Figures 6 and 7 are plotted with respect to the number of unknowns so that different basis functions can be compared fairly. However, since the LL and $\hat{\boldsymbol{n}} \times$ LL functions are defined in pairs at the edges, their triangulation is different from the triangulation of the RWG and $\hat{\boldsymbol{n}} \times$ RWG functions to keep the number of unknowns fixed. In other words, a coarser triangulation is employed for the LL and $\hat{\boldsymbol{n}} \times \mathrm{LL}$ functions. Employing these functions for a given number of unknowns, the number of triangles to model the geometry is about half of that for the RWG and $\hat{\boldsymbol{n}} \times$ RWG functions. (In fact, it is exactly half if the geometry is also closed, as discussed by Gürel et al. [1999].) For curved geometries, a coarser triangulation leads to a larger deviation from the actual geometry. Therefore, in the example of the sphere problem, we keep in mind that the LL and $\hat{\boldsymbol{n}} \times$ LL functions improve the accuracy in spite of the decreased quality of the geometry discretization.
[44] As another example, we consider the scattering problem of a perfectly conducting cube with edges of $d=$ $1 \mathrm{~m}$ as shown in Figure 8. The problem is solved at $300 \mathrm{MHz}(d=\lambda)$ and $600 \mathrm{MHz}(d=2 \lambda)$ with the same plane wave excitation as for the sphere problem. Mesh size of about $\lambda / 10$ leads to triangulations with 2052 and 8046 edges for $d=\lambda$ and $2 \lambda$, respectively. Figures 9 and 10 display the backscattered and forward-scattered RCS $\left(\mathrm{m}^{2}\right)$ values, plotted again with respect to the number of unknowns. As in the sphere problem, we observe faster convergence for the LL and $\hat{\boldsymbol{n}} \times$ LL functions, while the RWG function gives the worst accuracy for a given number of unknowns in the MFIE.

[45] To demonstrate the effect of using different basis functions on the modeling of the induced current, Figures 11 and 12 show the magnitude of the dominant $y$ component of the normalized surface current induced on the front surface (at $x=d / 2$ ) of perfectly conducting cubes with edges of $d=\lambda$ and $d=2 \lambda$, respectively. The modeled current is obtained for all four functions and triangulations with mesh size of about $\lambda / 10$. It is evident from Figures 11 and 12 that the modeling is significantly

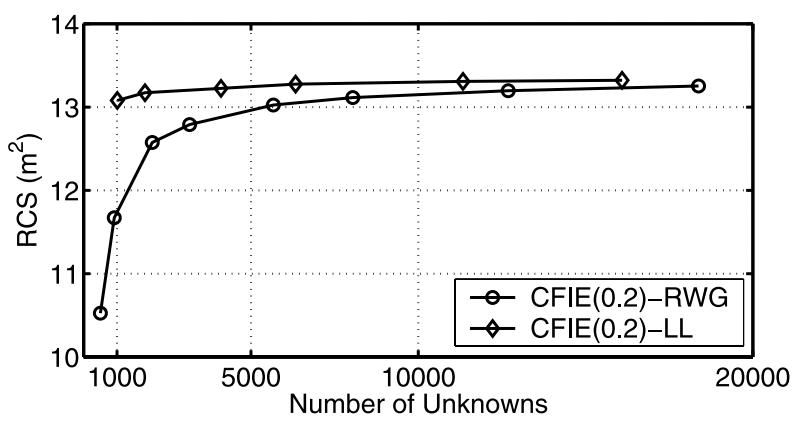

(a)

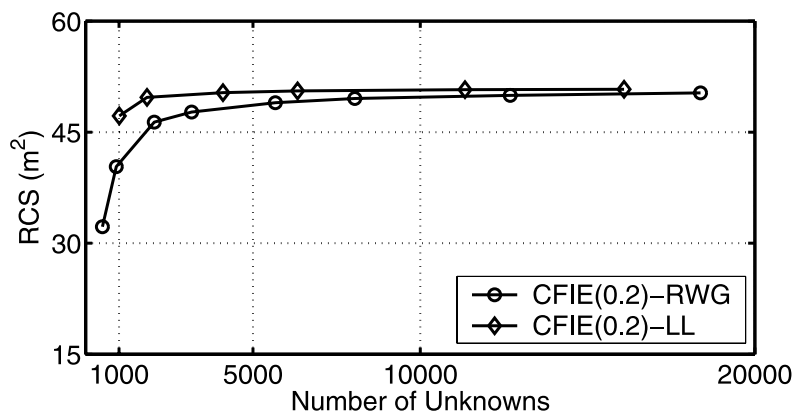

(b)

Figure 16. Backscattered RCS $\left(\mathrm{m}^{2}\right)$ of the perfectly conducting cube in Figure 8 with (a) $d=\lambda$ and (b) $d=2 \lambda$ with respect to the number of unknowns. 


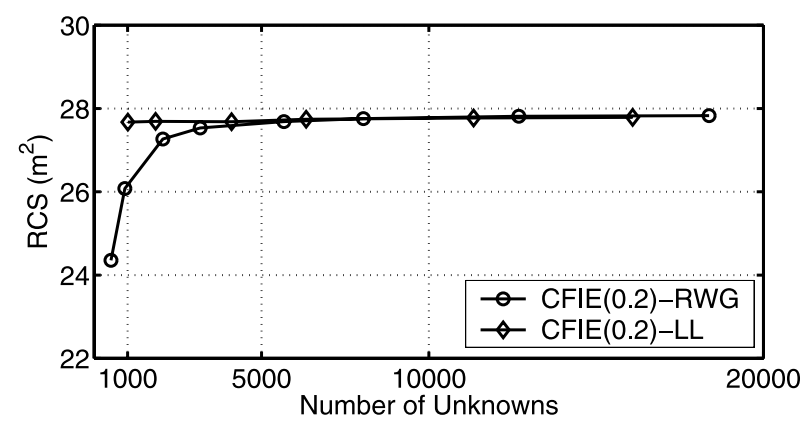

(a)

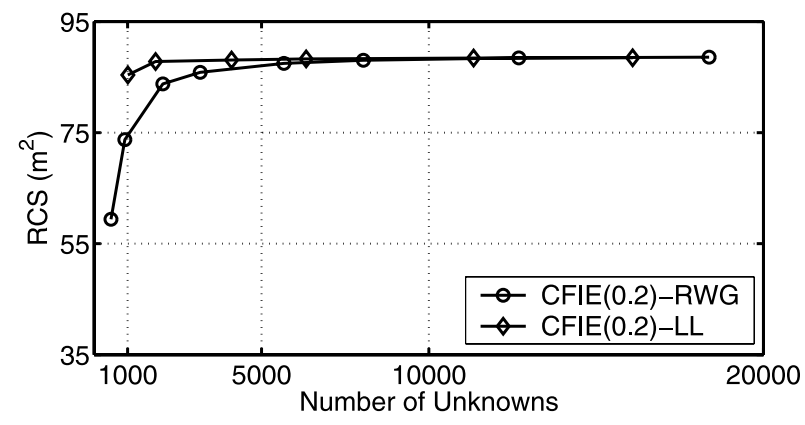

(b)

Figure 17. Forward-scattered $\operatorname{RCS}\left(\mathrm{m}^{2}\right)$ of the perfectly conducting cube in Figure 8 with (a) $d=\lambda$ and (b) $d=2 \lambda$ with respect to the number of unknowns.

improved with the LN-LT type functions compared to the CN-LT type RWG function and LN-CT type $\hat{\boldsymbol{n}} \times \mathrm{RWG}$ function, especially for the representation of singular current at the edges. We also note that the features and the quality of the current modeling are very similar for both the divergence-conforming LL functions and the curl-conforming $\hat{\boldsymbol{n}} \times$ LL functions; this observation is also consistent with the RCS results.

[46] To compare the RWG and LL functions in terms of the accuracy of the CFIE implementations, Figures 13 and 14 show the backscattered and forward-scattered RCS values, respectively, for the sphere problem in Figure 5 with $a=\lambda$ and $1.5 \lambda$ and a mesh size of about $\lambda / 10$. The RCS values are calculated for different values of the $\alpha$ in (17). Therefore the values at $\alpha=0$ and $\alpha=1$ correspond to those obtained by the MFIE and EFIE, respectively. Besides the overall improvement in accuracy by the use of the LL functions, Figures 13 and 14 demonstrate that the significant and undesired variance in accuracy with the use of the RWG functions is dramatically reduced with the use of the LL functions. In other words, accuracy is stabilized with respect to the variable $\alpha$ and becomes weakly dependent on the choice of this variable.
[47] Although there is no optimum $\alpha$ for the accuracy of the LL functions, Figure 15 shows that the conditioning of the matrix equation depends strongly on this variable. In Figure 15, conjugate gradient squared (CGS) iteration counts required to reach $10^{-3}$ residual error for the FMM solution of the sphere problem are depicted. The FMM employing the CGS solver is accelerated by a block diagonal preconditioner obtained by keeping the self-interactions of the clusters in the FMM structure. It is observed that the approximate range of $\alpha$ from 0.2 to 0.3 is optimum for both the RWG and LL functions.

[48] Finally, Figures 16 and 17 present the backscattered and forward-scattered RCS values for the cube problem in Figure 8 with $d=\lambda$ and $d=2 \lambda$, computed by using a CFIE implementation with $\alpha=0.2$. The CFIE formulations employing the RWG and LL functions are compared. In this convergence analysis, we also observe a significant improvement in the accuracy of the CFIE by using the LL functions.

\section{Conclusion}

[49] Comparisons of the linear basis functions for the MFIE and CFIE implementations show that accuracy can be improved significantly by using LN-LT type functions for current modeling on planar triangulations of arbitrary geometries. The accuracy problem, observed in previous studies [Ergül and Gürel, 2006], of the MFIE (and, consequently, the CFIE) can be eliminated in this way. Although the RWG functions are sufficient in terms of the accuracy of the EFIE, these functions are better to be replaced by the divergence-conforming LL functions in order to obtain MFIE and CFIE results that have the same levels of accuracy as the EFIE.

\section{Appendix A: Decomposition of RWG Functions Into LL Functions}

[50] In this appendix, we present the LL functions by following the notation of Graglia et al. [1997]. This convention is useful since it provides the mathematical background for the derivation of the LL functions from the RWG functions by a decomposition. A similar decomposition appeared in the literature to improve the accuracy of the 3-D finite element method [Mur and de Hoop, 1985].

[51] Graglia et al. [1997] give the zeroth-order divergence-conforming bases for the triangular elements, such as the RWG functions, as

$$
\boldsymbol{\Lambda}_{\beta}(\boldsymbol{r})=\frac{1}{J_{\beta}}\left[\xi_{\beta+1}(\boldsymbol{r}) \boldsymbol{\ell}_{\beta-1}-\xi_{\beta-1}(\boldsymbol{r}) \boldsymbol{\ell}_{\beta+1}\right], \quad \beta=1,2,3,
$$


where $J_{\beta}$ indicates the Jacobian derived for the RWG functions as

$$
J_{\beta}=\frac{2 A}{\left|\boldsymbol{\ell}_{\beta}\right|}
$$

and $A$ is the area of the triangle. In (A1), $\xi_{i}(\boldsymbol{r})$ for $i=1,2$, 3 represent the parent coordinates with the dependency relationship

$$
\xi_{1}(\boldsymbol{r})+\xi_{2}(\boldsymbol{r})+\xi_{3}(\boldsymbol{r})=1
$$

when $\boldsymbol{r}$ is on the triangle. In addition, $\ell_{i}$ in (A1) for $i=1$, 2,3 represent the edge vectors with the dependence

$$
\ell_{1}+\ell_{2}+\ell_{3}=0
$$

[52] RWG functions, which provide three DOFs per triangle, are zeroth-order complete since they can represent any constant vector function on a triangle and their surface divergence is also constant. Given the three RWG functions corresponding to the three edges of a triangle as in (A1), the set of LL functions associated with the same triangle can be derived as

$$
\begin{gathered}
\boldsymbol{\Lambda}_{\boldsymbol{\beta}}^{(1)}(\boldsymbol{r})=\frac{1}{J_{\beta}} \xi_{\beta+1}(\boldsymbol{r}) \boldsymbol{\ell}_{\beta-1} \\
\boldsymbol{\Lambda}_{\boldsymbol{\beta}}^{(2)}(\boldsymbol{r})=-\frac{1}{J_{\beta}} \xi_{\beta-1}(\boldsymbol{r}) \boldsymbol{\ell}_{\beta+1}
\end{gathered}
$$

with the decomposition

$$
\boldsymbol{\Lambda}_{\boldsymbol{\beta}}(\boldsymbol{r})=\boldsymbol{\Lambda}_{\boldsymbol{\beta}}^{(1)}(\boldsymbol{r})+\boldsymbol{\Lambda}_{\boldsymbol{\beta}}^{(2)}(\boldsymbol{r}), \quad \beta=1,2,3 .
$$

We note that both of the distributions in (A1) and (A5)(A6) provide a linear variation for the parallel components along the edges $\beta$. On the other hand,

$$
\begin{aligned}
& \left.\nabla \xi_{\beta}(\boldsymbol{r})\right|_{\xi_{\beta}(\boldsymbol{r})=0} \cdot \boldsymbol{\Lambda}_{\beta}^{(1)}(\boldsymbol{r})=\xi_{\beta+1}(\boldsymbol{r}) \\
& \left.\nabla \xi_{\beta}(\boldsymbol{r})\right|_{\xi_{\beta}(\boldsymbol{r})=0} \cdot \boldsymbol{\Lambda}_{\beta}^{(2)}(\boldsymbol{r})=\xi_{\beta-1}(\boldsymbol{r})
\end{aligned}
$$

so that the normal components of the LL functions in (A5)-(A6) have also linear variation along the edges $\beta$, while they are constant for the RWG functions in (A1), that is,

$$
\begin{aligned}
& \left.\nabla \xi_{\beta}(\boldsymbol{r})\right|_{\xi_{\beta}(\boldsymbol{r})=0} \cdot \boldsymbol{\Lambda}_{\boldsymbol{\beta}}(\boldsymbol{r})=\xi_{\beta+1}(\boldsymbol{r})+\xi_{\beta-1}(\boldsymbol{r}) \\
& \quad=1-\xi_{\beta}(\boldsymbol{r})=1 .
\end{aligned}
$$

[53] Any linearly varying vector function on the triangle can be represented by a combination of the LL functions since

$$
\begin{gathered}
\frac{\Lambda_{2}^{(1)}(\boldsymbol{r})}{\left|\boldsymbol{\ell}_{2}\right|}+\frac{\boldsymbol{\Lambda}_{2}^{(2)}(\boldsymbol{r})}{\left|\boldsymbol{\ell}_{2}\right|}-\frac{\Lambda_{3}^{(1)}(\boldsymbol{r})}{\left|\boldsymbol{\ell}_{3}\right|}-\frac{\Lambda_{3}^{(2)}(\boldsymbol{r})}{\left|\boldsymbol{\ell}_{3}\right|}=\frac{\boldsymbol{\ell}_{1}}{2 A} \\
\frac{\boldsymbol{\Lambda}_{3}^{(1)}(\boldsymbol{r})}{\left|\boldsymbol{\ell}_{3}\right|}+\frac{\boldsymbol{\Lambda}_{3}^{(2)}(\boldsymbol{r})}{\left|\boldsymbol{\ell}_{3}\right|}-\frac{\Lambda_{1}^{(1)}(\boldsymbol{r})}{\left|\boldsymbol{\ell}_{1}\right|}-\frac{\boldsymbol{\Lambda}_{1}^{(2)}(\boldsymbol{r})}{\left|\boldsymbol{\ell}_{1}\right|}=\frac{\boldsymbol{\ell}_{2}}{2 A} \\
\frac{\boldsymbol{\Lambda}_{2}^{(2)}(\boldsymbol{r})}{\left|\boldsymbol{\ell}_{2}\right|}-\frac{\boldsymbol{\Lambda}_{3}^{(1)}(\boldsymbol{r})}{\left|\boldsymbol{\ell}_{3}\right|}=\frac{\xi_{1}(\boldsymbol{r}) \boldsymbol{\ell}_{1}}{2 A} \\
\frac{\boldsymbol{\Lambda}_{3}^{(2)}(\boldsymbol{r})}{\left|\boldsymbol{\ell}_{3}\right|}-\frac{\boldsymbol{\Lambda}_{1}^{(1)}(\boldsymbol{r})}{\left|\boldsymbol{\ell}_{1}\right|}=\frac{\xi_{2}(\boldsymbol{r}) \boldsymbol{\ell}_{2}}{2 A} \\
\frac{\boldsymbol{\Lambda}_{3}^{(2)}(\boldsymbol{r})}{\left|\boldsymbol{\ell}_{3}\right|}=\frac{\xi_{2}(\boldsymbol{r}) \boldsymbol{\ell}_{1}}{2 A} \\
\mid \boldsymbol{\ell}_{3}^{(1)}(\boldsymbol{r}) \\
=\frac{\xi_{1}(\boldsymbol{r}) \boldsymbol{\ell}_{2}}{2 A}
\end{gathered}
$$

On the other hand, the LL functions are not strictly firstorder complete since their surface divergences

$$
\nabla_{S} \cdot \Lambda_{\beta}^{(1)}(\boldsymbol{r})=\frac{1}{J_{\beta}} \boldsymbol{\ell}_{\beta-1} \cdot \nabla \xi_{\beta+1}(\boldsymbol{r})=\frac{1}{J_{\beta}}=\frac{\left|\boldsymbol{\ell}_{\beta}\right|}{2 A}
$$

$$
\nabla_{S} \cdot \Lambda_{\beta}^{(2)}(\boldsymbol{r})=-\frac{1}{J_{\beta}}(\boldsymbol{r}) \boldsymbol{\ell}_{\beta+1} \cdot \nabla \xi_{\beta-1}(\boldsymbol{r})=-\frac{1}{J_{\beta}}=-\frac{\left|\boldsymbol{\ell}_{\beta}\right|}{2 A}
$$

for $\beta=1,2$, or 3 are constants. As indicated by Graglia et al. [1997], a strictly first-order complete basis requires eight DOFs on the triangle, whereas the LL functions have six DOFs. Nevertheless, LL functions have the advantage that their implementations do not require higher-order techniques while providing significantly higher accuracy than the RWG functions. Furthermore, most of the details of their implementations, such as the singularity extraction for the near-field interactions, can be derived from those of the RWG functions, thereby facilitating their numerical implementations. Finally, LL functions can also be viewed as a subset of the QN/LT or 
LN/QT type vector functions discussed by Peterson et al. [1998].

[54] Acknowledgments. The authors would like to thank the anonymous reviewers for their great efforts and invaluable suggestions to improve the manuscript. This work was supported by the Turkish Academy of Sciences in the framework of the Young Scientist Award Program (LG/TUBA-GEBIP/ 2002-1-12), by the Scientific and Technical Research Council of Turkey (TUBITAK) under research grant 105E172, and by contracts from ASELSAN and SSM.

\section{References}

Chew, W. C., J.-M. Jin, E. Michielssen, and J. Song (2001), Fast and Efficient Algorithms in Computational Electromagnetics, Artech House, Norwood, Mass.

Coifman, R., V. Rokhlin, and S. Wandzura (1993), The fast multipole method for the wave equation: A pedestrian prescription, IEEE Antennas Propag. Mag., 35, 7-12.

Ergül, Ö., and L. Gürel (2004a), Investigation of the inaccuracy of the MFIE discretized with the RWG basis functions, in IEEE Antennas and Propagation Society International Symposium 2004, vol. 3, pp. 3393-3396, IEEE Press, Piscataway, N. J.

Ergül, Ö., and L. Gürel (2004b), Improving the accuracy of the MFIE with the choice of basis functions, in IEEE Antennas and Propagation Society International Symposium 2004, vol. 3, pp. 3389-3392, IEEE Press, Piscataway, N. J.

Ergül, Ö., and L. Gürel (2005a), Improved testing of the magnetic-field integral equation, IEEE Microwave Wireless Components Lett., 15, 615-617.

Ergül, Ö., and L. Gürel (2005b), Solid-angle factor in the magnetic-field integral equation, Microwave Opt. Technol. Lett., 45, 452-456.

Ergül, Ö., and L. Gürel (2006), The use of curl-conforming basis functions for the magnetic-field integral equation, IEEE Trans. Antennas Propag., 54, 1917-1926.

Glisson, A. W., and D. R. Wilton (1980), Simple and efficient numerical methods for problems of electromagnetic radiation and scattering from surfaces, IEEE Trans. Antennas Propag., 28, 593-603.

Graglia, R. D. (1993), On the numerical integration of the linear shape functions times the 3-D Green's function or its gradient on a plane triangle, IEEE Trans. Antennas Propag., 41, $1448-1455$.

Graglia, R. D., D. R. Wilton, and A. F. Peterson (1997), Higher order interpolatory vector bases for computational electromagnetics, IEEE Trans. Antennas Propag., 45, $329-342$.

Gürel, L., and Ö. Ergül (2003), Comparisons of FMM implementations employing different formulations and iterative solvers, in IEEE Antennas and Propagation Society International Symposium 2003, vol. 1, pp. 19-22, IEEE Press, Piscataway, N. J.
Gürel, L., and Ö. Ergül (2005), Singularity of the magneticfield integral equation and its extraction, IEEE Antennas Wireless Propag. Lett., 4, 229-232.

Gürel, L., K. Sertel, and I. K. Şendur (1999), On the choice of basis functions to model surface electric current densities in computational electromagnetics, Radio Sci., 34, 13731387.

Harrington, R. F. (1968), Field Computation by Moment Methods, Macmillan, New York.

Hodges, R. E., and Y. Rahmat-Samii (1997), The evaluation of MFIE integrals with the use of vector triangle basis functions, Microwave Opt. Technol. Lett., 14, 9-14.

Lu, C.-C., and W. C. Chew (1997), Multilevel fast multipole algorithm for electromagnetic scattering by large complex objects, IEEE Trans. Antennas Propag., 45, 1488-1493.

Mautz, J. R., and R. F. Harrington (1978), $H$-field, $E$-field, and combined field solutions for conducting bodies of revolution, Arch. Elektron. Uebertraeg., 32, 157-164.

Morita, N., N. Kumagai, and J. R. Mautz (1990), Integral Equation Methods for Electromagnetics, Artech House, Norwood, Mass.

Mur, G., and A. T. de Hoop (1985), A finite-element method for computing three-dimensional electromagnetic fields in inhomogenous media, IEEE Trans. Magn., 21, 21882191.

Peterson, A. F. (2002), Solution of the MFIE using curl-conforming basis functions, in IEEE Antennas and Propagation Society International Symposium 2002, vol. 1, pp. 70-73, IEEE Press, Piscataway, N. J.

Peterson, A. F., S. L. Ray, and R. Mittra (1998), Computational Methods for Electromagnetics, IEEE Press, Piscataway, N. J.

Poggio, A. J., and E. K. Miller (1973), Integral equation solutions of three-dimensional scattering problems, in Computer Techniques for Electromagnetics, edited by R. Mittra, chap. 4, pp. 159-261, Elsevier, New York.

Rao, S. M., and D. R. Wilton (1990), $E$-field, $H$-field, and combined field solution for arbitrary shaped threedimensional dielectric bodies, Electromagnetics, 10, 407-421.

Rao, S. M., D. R. Wilton, and A. W. Glisson (1982), Electromagnetic scattering by surfaces of arbitrary shape, IEEE Trans. Antennas Propag., 30, 409-418.

Rius, J. M., E. Úbeda, and J. Parrón (2001), On the testing of the magnetic field integral equation with RWG basis functions in method of moments, IEEE Trans. Antennas Propag., 49, 1550-1553.

Shanker, B., A. A. Ergin, K. Aygün, and E. Michielssen (2000), Analysis of transient electromagnetic scattering from closed surfaces using a combined field integral equation, IEEE Trans. Antennas Propag., 48, 1064-1073.

Sheng, X.-Q., J.-M. Jin, J. Song, W. C. Chew, and C.-C. Lu (1998), Solution of combined-field integral equation using multilevel fast multipole algorithm for scattering by homogeneous bodies, IEEE Trans. Antennas Propag., 46, 17181726. 
Song, J. M., C. C. Lu, W. C. Chew, and S. W. Lee (1998), Fast Illinois solver code (FISC), IEEE Antennas Propag. Mag., 40, 27-34.

Trintinalia, L. C., and H. Ling (2001), First order triangular patch basis functions for electromagnetic scattering analysis, J. Electromagn. Waves Appl., 15, 1521-1537.

Wang, J., and J. P. Webb (1997), Hierarchal vector boundary elements and p-adaptation for 3-D electromagnetic scattering, IEEE Trans. Antennas Propag., 45, 1869-1879.

Ylä-Oijala, P., and M. Taskinen (2003), Calculation of CFIE impedance matrix elements with RWG and $\hat{\boldsymbol{n}} \times \mathrm{RWG}$ functions, IEEE Trans. Antennas Propag., 51, $1837-$ 1846.

Zhang, Y., T. J. Cui, W. C. Chew, and J.-S. Zhao (2003), Magnetic field integral equation at very low frequencies, IEEE Trans. Antennas Propag., 51, 1864-1871.

Ö. Ergül and L. Gürel, Department of Electrical and Electronics Engineering, Bilkent University, TR-06800, Bilkent, Ankara, Turkey. (ergul@ee.bilkent.edu.tr; lgurel@ bilkent.edu.tr) 